\section{THE TREATMENT OF TRICHIASIS AND ENTROPION.}

BY W. A. MCKEOWN, M.D., M.CI.,

SURGEON TO THE ULSTER EYE, EAR, AND THROAT HOSPITAL, BELFAST.

THE unsatisfactory nature of the treatment of these affections may be judged by the large number of operative procedures of one sort or other which the writers of hand. books of diseases of the eye think it necessary to detail. There can be no question about the ingenuity displayed, but as a rule it is not directed to simplicity of method. I find in Swanzy's excellent handbook no less than eight chief operations for trichiasis and organic entropion and four for spastic entropion. In Meyer's book there are about a score of operations described and more mentioned. These books purport to be written chiefly for students and general practitioners, but even to one famillar with oph thalmic surgery it requires abtentive reading to grasp all the technicalities. The operations referred to may be objected to on one or more grounds. First, unnecessary destruction of tissue; secondly, complexity and difficulty in execution; and, thirdly, uncerbainty. I will not discuss or even name these different operations, but proceed to describe a method which I have practised extensively, and which I think will supplant all other methods. The operation is as follows. First stage: If the upper lid is to be operated on, introduce the smooth handle of a dessertspoon under the lid, to protect the eyeball and support the lid ; let the assistant hold it whilst the surgeon steadies the lid on the handle with the left hand, and with the right hand introduces a Graefe's cataract knife at the margin of the lid between the eyelashes and the Meibomian glands, either close to the punctum or at the outer canthus; then push the knife beneath the skin about as far as the orbital margin, and by to-and-fro motion cut along the whole of the lid margin until he has the lid split into two layers, the outer layer containing the skin and eyelashes, the inner layer the cartilage, Meibomian glands, \&c. If the skin and hairs can be moved freely upwards, the section has been properly made; if not, blunt-pointed scissors should be passed into the sestion, and any tissue preventing frce motion cut. Second stage : The skin and eyelashes are glided upwards so as to expose about two lines of the cartilage, and sire to be secured in their new position as follows. A curved needle with a strong silk iodoformed thread is to be passed through the skin at the middle of the lid abont two lines from the eyelashes, then through the cartilage, emerging on to the conjunctival surface about four lines from the margin, and then the needle is to be passed back again through the cartilage and skin, coming out a line or so from the original point of entry on the same horizontal line. A thread is then passed in the same way near the outer and inner canthus respectively. The skin layer and the cariloginous layer of the lid are now secured by three loops of thread in such a way that the lashes must be dieplaced up wards far from the eye. Each thread is tied with moderate tightness over a tiny roll of lint or cotton-wool. A dhesinn of the two layers in the new position quickly rakes place. It is evident that at the outer and inner corners of the section the hairs will not be so much deviated as an the centre, and if the surgeon should think there was any special tendency to a tarning in of the hairs at these parts he should make a small vertical incision through the skin, and by a suture at each corner elevate the eyelashes to the desired extent. Third stage (grafting): At one time I followed von Milligen's method by taking a graft from the mucous membrane of the lip, but I found that the taking of euch a graf was not a very expeditions procedure. I have for a considerable time taken the graft from the back of the ear, and for ease of performance and perfection in result I do not think it could be surpassed. I expose the back surface of the ear, pass a Graefe's knife under the skin for about an inch, then cut out on one side, and separate the attached side by fine scissors. I place the graft on the back of my lefo hand, and with fine scissors remove all subcutaneous tissue (this can be done quickly); remove any blood with lint soaked in warm boracic acid lotion, and immediately apply the grafo to the exposed surface of the tarsal cartilage, previously cleared of all clots, and likewise cleansed with boracic lotion.
I may remark that I think solution of perchloride of mercury not desirable in grafting operations because of ito coagulating power. I adjust carefully the graft by a probe, and do not proceed to the dressing until it seems to be prebty adherent. Fourth stage (the dressing): Apply a little strip of lint, saturated with warm iodoform and vaseline ointment, over the graft, and on the top of that a piece of lint to cover the whole eyelid, similarly saturated, then dry lint and bandage. Both eyes are bandaged. The patient is put to bed and not disturbed for four days. At the lapse of that time the stitches are removed, but the eye operated on is bandaged for three or four days more. It may be observed that I couple trichiasis and entropion, and write of them as if to be treated by one operation. If the cartilage be thickened or distorted, it is obvious that with such a free exposure of the cartilage the surgeon can do anything with it; he may groove it or pare \&c. as surgeons have recommended, but the grooving, paring, \&c., would be only a small incident in the general operation, and hardly worth naming as a separate or moditied method.

The features of this operation are: I. That no skin or other tissue is destroyed or removed, save in thickening or distortion of the cartilage. 2. Expedition in performance. 3. Certainty of immediate result, and no liability to relapse. 4. Its applicability to all sorts of trichissis and organic entropion. I have not described the operation for trichiasis of the lower lid, as there is but slight variation. As to what is called "spastic entropion" of the lower lid in old persons, arising chiefly from relaxation of the skin, I see in the text-books, old and new, several operations described. The trouble, however, may be sufficiently relieved without any operation at all. All that is necessary is to put a little pressure on the lower lid, and that may be conveniently done by a spectacle-frame of such a form es to press on the lower lid, or, if that cannot be found, by putting a ledge on the lower edge of a frame. For this suggestion I am in. debted to my brother, Dr. McKeown of Manchester, who has already put it in practice. Patients prefer this simple and effectual method to any operation. When only a few hairs are deviated I see in some books of repute that it is recommended to excise the hairs with the skin. I have never done this, but I have seen cases in which it was done with disastrous results. Even when a few hairs are fauloy it is commonly better to operate on the whole lid, as I have described. It seems a more formidable operation, but in the matter of personal appearance it is far zuperior to the seemingly simple excision. Should I wish to get rid of a few hairs and not to do the complete lid-splitting, I introduce the knife between the faulty hairs and the Meibomian glands, bring the point out through the skin two or three lines from the margin, then cut on till I have passed all the hairs to be dealt with, and cut out on the skin surface. I thus have a libtle flap which I can evert. I snip out the roots of the faulty hairs, then replace the little flap, and secure it by suture. Neither skin nor margin of the lid is injured. Belfast, Ireland.

\section{ATTEMPTED POISONING BY CARBOLIC ACID.}

BY E. C. KINGSFORD, L.R.C.P.LOND., M R.C.S.

THE following case of attempted poisoning by carbolic acid is, perhaps, of even greater interest than that reported by Dr. Greenway in The LANCET of Aug. 29th, 1891, p. 485, in that the acid was not diluted, and that no treatment was attempted till more than seven hours after the "accident."

J. G_, aged thirty-six, was admitted to the Bolton Infirmary at 2 P M. on Aug. 22nd, 1889, under the care of Mr. G. A. Patrick, with a report that he had poisoned himself with carbolic acid. He was quite unconscious, but rolling about as if in great agony, and breathing in an irregular and spasmodic manner, each expiratory effort being accompanied by a loud bellowing noise; his extremities and surface were cold and bathed in a clammy perspiration, and the pulse could scarcely be felt anywhere. The tongue and inside of the mouth were covered with a thick white slough, and the skin from the right corner of the mouth to the chin was excoriated where some of the fluid had trickled down. Ineffectual attempts were made to force him to swallow 